\title{
Lower urinary-tract symptoms and testosterone in elderly men
}

\author{
Aksam A. Yassin · Ahmed I. El-Sakka • Farid Saad • \\ Louis J. G. Gooren
}

Received: 3 March 2008 / Accepted: 19 May 2008 / Published online: 2 July 2008

(C) The Author(s) 2008

\begin{abstract}
Objectives The objective was to examine the effects of testosterone administration on symptom scores of lower urinary tract symptoms (LUTS).

Methods The literatures on the epidemiological association between the metabolic syndrome, erectile failure and (LUTS) were reviewed.

Results In men with the metabolic syndrome and erectile failure, often lower-than-normal testosterone levels are found. This is less clear for men with LUTS, but the relationship between testosterone and LUTS might be indirect and based on the association of the metabolic syndrome with an overactivity of autonomic nervous system. This
\end{abstract}

\section{A. A. Yassin}

Institute of Urology and Andrology,

Segeberger Kliniken, Norderstedt, Germany

A. A. Yassin · F. Saad

Gulf Medical University School of Medicine, Ajman, UAE

A. I. El-Sakka

Department of Urology, Suez Canal University,

School of Medicine, Ismailia, Egypt

\section{A. I. El-Sakka}

Al-Noor Specialist Hospital, Makkah, Saudi Arabia

F. Saad

Bayer Schering Pharma,

Scientific Affairs Men's Healthcare, Berlin, Germany

L. J. G. Gooren

Endocrinology, VUMC, Amsterdam, The Netherlands

e-mail: ljgooren@truemail.co.th

\section{A. A. Yassin $(\square)$}

Rathausallee 94a, 22846 Norderstedt, Hamburg, Germany

e-mail: yassin@t-online.de overactivity may play a key role in increasing the severity of LUTS above an intrinsic basal intensity that is determined by the genitourinary factors in aging men. Androgen receptors are present in the epithelium of the urethra and the bladder. Testosterone may play a role in the reflex activity of the autonomic nervous system in the pelvis, or may interact with postsynaptic non-genomic receptors suppressing detrusor activity. Human neurons in the wall of the bladder contain nitric oxide synthase. Similar to the penis, testosterone has an impact on nitric oxide synthase.

Conclusions Some studies investigating the effects of normalizing testosterone levels in elderly men have found a positive effect on variables of the metabolic syndrome and, simultaneously, on scores of the International Prostate Symptoms Score (IPSS) which is worthy of further investigation in randomized, controlled and sufficiently powered clinical trials.

Keywords Lower urinary-tract symptoms · Aging ·

Testosterone $\cdot$ Phospho-diesterase type 5

\section{Introduction}

Over the past two decades, it becomes clear that many agerelated health problems of men, hitherto treated by different medical disciplines, are actually inter-related and require a more integrative approach to the health situation of the aging male. At the epidemiological level, an association between central obesity in adulthood, the metabolic syndrome, erectile failure and lower urinary-tract symptoms (LUTS) has been established [1]. A common denominator of the above ailments is lower-than-normal testosterone levels occurring in a significant proportion of elderly men [2]. The decline of serum testosterone may be demonstrable over relatively short periods of observation. Throughout a 
4-year follow-up in elderly patients with erectile dysfunction, there was a steady decrease in testosterone levels [3].

Many studies have tried to establish a relationship between sex steroids and benign prostate hyperplasia, and a few studies have analyzed the relationship between circulating testosterone and LUTS symptoms. One study found that hypogonadism was seen in approximately one-fifth of elderly men with LUTS, but it had no impact on symptom status [4]. Another study found a relationship between symptoms of LUTS and plasma total and bioavailable testosterone but this relationship disappeared after statistical adjustment for age [5]. No consistent correlations were found between total and calculated free testosterone and symptoms of LUTS in another study, but there was a relationship with androstanediolglucuronide, a metabolite of dihydrotestosterone and with estradiol [1]. A very recent study found a relationship between circulating androgens and bladder outlet obstruction. Low $\mathrm{T}$ levels in clinical bladder outlet obstruction correlated negatively with detrusor pressure at maximum flow and at urethral closure while promoting detrusor overactivity [6].

Overall it has been difficult to relate plasma testosterone to LUTS, but it is of note that, within certain limits of testosterone levels, the signs and symptoms of testosterone deficiency in men do not relate in a uniform pattern to testosterone concentrations [7] which may be (in part) explained by properties of the androgen receptor (the CAG repeat polymorphism in exon 1 of the androgen receptor gene) [8]. Nevertheless, it must be concluded that so far no clear relationship between LUTS and testosterone could be demonstrated. It could well be that the detrimental effects of testosterone deficiency and the benefits of testosterone treatment are indirect. The following sections will discuss the various elements of the urodynamic system on which $\mathrm{T}$ impacts. This is graphically presented in Fig. 1. Whether these elements are interrelated remains to be determined.

\section{The relationship between the metabolic syndrome and LUTS}

A recent study trying to explain the epidemiological relationship between the metabolic syndrome and LUTS hypothesized that the metabolic syndrome is associated with an overactivity of the autonomic nervous system [9, 10] for which hyperinsulinemia, a key element of the metabolic syndrome might be responsible $[9,10]$. This overactivity of the autonomic nervous system is supposedly not responsible for the development of LUTS, but plays a key role in increasing the severity of LUTS above an intrinsic basal intensity that is determined by the genitourinary anatomical/pathophysiological characteristics of other ailments leading to LUTS $[11,12]$. Another recent study provided evidence that stress conditions could be associated with the development and aggravation of prostatic disease. It was found that body mass index, and age, greater diastolic blood pressure reactivity were associated with a greater transition zone volume, greater total prostate gland volume, greater postvoid residual bladder volume, and more severe LUTS [13]. Inflammatory infiltrates are frequently found in and around nodules in benign prostate hyperplasia (BPH) and in symptomatic BPH [14]. The presence of the metabolic syndrome might be a mediator of this association because it is associated with elevated serum $\mathrm{C}$-reactive protein concentration, a non-specific marker of inflammation [15], thus linking the metabolic syndrome to LUTS and
Fig. 1 Potential sites of action of testosterone on the urinary tract

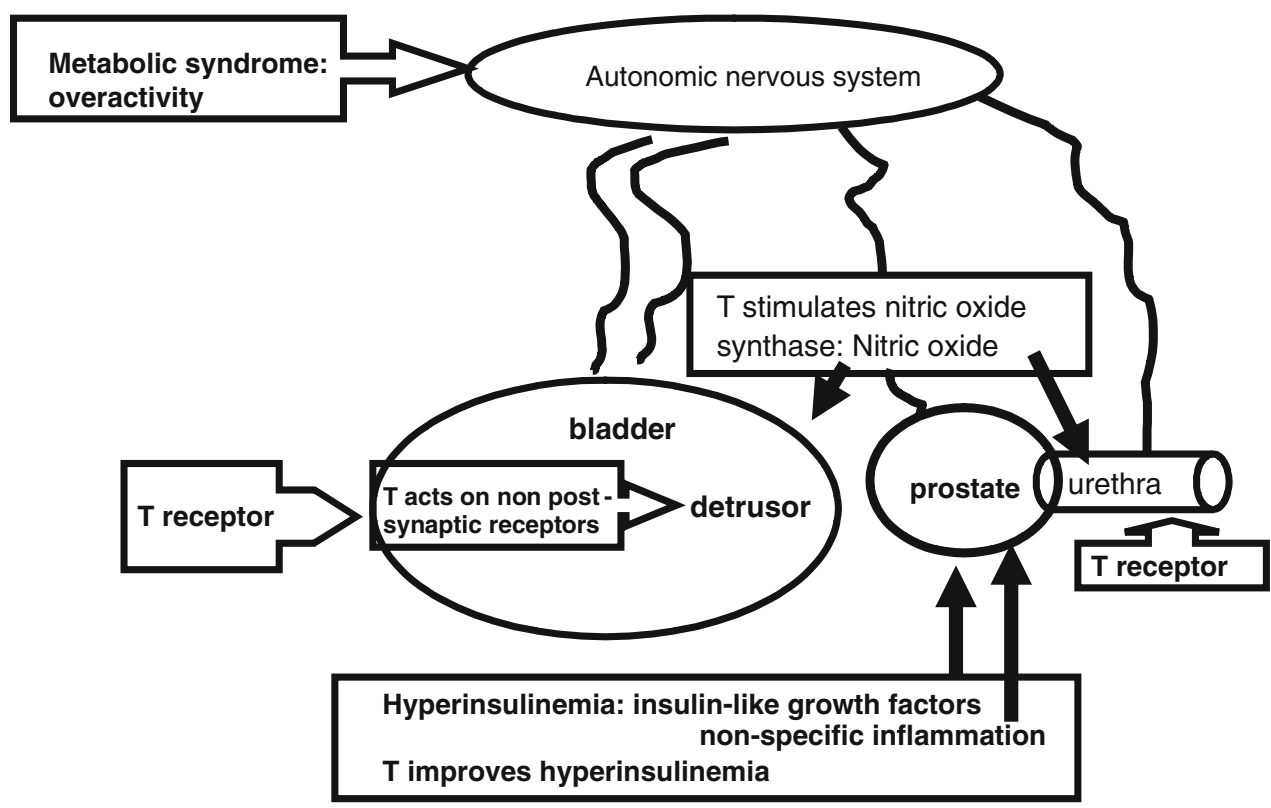


elevated circulating C-reactive protein concentrations might be an indicator of intraprostatic inflammation in symptomatic BPH $[14,15]$. (Central) obesity is a hallmark of the metabolic syndrome of which the other components are dyslipidemia, hypertension, impaired glucose metabolism, with insulin resistance and diabetes type 2. Particularly if poorly controlled, there is a significant association between low level of total testosterone or DHEA-S and indices of poorly controlled type 2 diabetes [16].

Insulin resistance is associated with hyperinsulinemia and insulin, particularly in excess, has due to its biochemical similarities with insulin-like growth factor, growth promoting properties [17]. This might be a factor in the growth of the prostate in aging men [18]. It has become clear that normal levels of T improve insulin resistance [19].

All the above-mentioned elements of the metabolic syndrome are conducive to the development not only of erectile dysfunction but also of LUTS. Risk factors and medical co-morbidities of erectile dysfunction were prevalent among patients with LUTS [20] (El-Sakka 2006b) and it is, therefore, not surprising that a larger number of studies have established a relationship between LUTS and erectile dysfunction [21-23], particularly since an underlying vascular association between LUTS and erectile dysfunction could be demonstrated [24].

As indicated earlier, with a more integrative approach to the ailments of the aging male, the age-related decline of plasma testosterone levels has been found to be a feature of erectile failure and central obesity in elderly men with proven successes of administration of testosterone to correct lower-than-normal levels [25-29]. So, it is time to review the relationship between late onset hypogonadism $(\mathrm{LOH})$ and LUTS, which like the other ailments mentioned earlier, manifest themselves concurrently in the lives of elderly men.

\section{Potential effects of testosterone on the urinary tract}

Testosterone itself might not be the 'prime mover' of the effects of testosterone on those structures of the urinary tract anatomically and functionally related to LUTS. The indirect relation could obscure an interrelation between circulating levels of testosterone and symptoms of LUTS at a statistically significant level which nevertheless is biologically plausible.

Androgen receptors have been found to be present to a large extent in the epithelial cells of the urethra and the bladder [30]. In another study, the role of testosterone and its metabolites on maintaining the reflex activity in the pelvic part of the autonomic nervous system could be demonstrated in rats [31]. Others have postulated the influence of testosterone on postsynaptic non-genomic receptors which are suppressing detrusor activity [32, 33]. Castration resulted in significant alterations in the activities of citrate synthase-thapsigargin sensitive $\mathrm{Ca}(2+)$ ATPase [sarco/ endoplasmic reticulum $\mathrm{Ca}(2+)$ ATPase (SERCA)], and choline acetyl-transferase as markers for mitochondrial function, sarcoplasmic reticular calcium storage and release, and cholinergic nerve function, in the bladder body, urethra, and corpora [34].

Not only the penis but also in other parts of the urogenital tract nitric oxide (NO) acts as a non-adrenergic non-cholinergic neurotransmitter in the urogenital tract and the action of testosterone on the urogenital tract may be mediated by this system [35]. There is increasing evidence for a link between ED and LUTS, the metabolic syndrome, pelvic atherosclerosis with its associated rho-kinase activation/ endothelin pathway, the NOS/NO theory, and the autonomic hyperactivity [36]. Studies treating one condition (e.g., ED) and measuring the impact on the other (e.g., LUTS) should further contribute to support this common link. But as yet it is not possible to provide a comprehensive picture of the impact of testosterone (and its deficiency) on the lower urinary tract.

\section{Nitric oxide production is androgen dependent in urinary tract}

Nitric oxide (NO) acting as a non-adrenergic non-cholinergic neurotransmitter is not only present in genital structures but also in the urinary tract and exerts a smooth muscle relaxing effect in both animals and humans. NO is a mediator not only of erection but also of dilatation of the bladder neck and urethra. A study in the human indicated that NO is an important nerve-induced mediator of erection and the micturition reflex, but NO may also be involved in several other functions in the human urogenital tract [37]. In humans, 72-96\% of neurons in the wall of the bladder appear to contain nitric oxide synthase. Nitric oxide synthase-immunoreactive nerve terminals provide a moderate innervation to the detrusor muscle of the bladder body, and a denser innervation to the urethral muscle. Nitric oxide may be an inhibitory transmitter involved in the relaxation of the bladder neck [38]. Cyclic nucleotides are important secondary messengers of nitric oxide involved in modulating the contractility of various smooth muscles.

Phosphodiesterases (PDE) play important roles in this process by modulating the levels of cyclic nucleotides and their duration of action. Their presence in the urinary bladder could be identified in studies of the rat [39] and the human [40].

Phosphodiesterase 5 is an inhibitor of nitric oxide/cGMP signaling. A recent study, investigating PDE5 expression and activity in the human bladder, elegantly demonstrated 
that PDE5 regulates smooth muscle tone of the bladder. Vardenafil appeared to block PDE5 activity, and therefore, may be a possible therapeutic option for bladder dysfunction by ameliorating irritative lower urinary-tract symptoms. The study also found that castration decreased, and testosterone supplementation restored, PDE5 gene expression in rat bladder [35].

As a further substantiation of the role of androgens in the urogenital tract, NO-synthase in an earlier study had appeared to be androgen dependent in the urogenital tract of the rat [41]. Meanwhile a large number of clinical studies have convincingly shown that phosphodiesterase inhibitors have a beneficial effect on LUTS [36, 42-48].

From the above, it would appear that androgens are not only essential for the formation of a male urogenital tract prenatally and its adult development during puberty but that, similar to erectile tissue in the penis, maintenance of the functionality of the urinary tract in adult life is subserved by androgens. It could be that declining testosterone production with aging contributes to the discomfort elderly men experience with micturition.

\section{Effects of testosterone on LUTS}

The first mention of effects of testosterone on bladder function was reported by Holmang et al. [49] finding an increase in peak urinary flow and mean urine volume voided in a testosterone-treated group of men compared to placebo treatment. But, in recent years there has been only preliminary evidence that men with LUTS benefit from treatment with testosterone, only in the form of abstracts awaiting peer-reviewed publication. The first data on this subject have shown that normalization of testosterone levels has a positive effect on LUTS in men with BPH and $\mathrm{LOH}$ [50]. A recent presentation confirmed the positive effects of testosterone treatment on bladder functions by increasing bladder capacity and compliance and decreasing detrusor pressure at maximal flow in men with SLOH [51]. The results of another pilot study [52] also showed positive effect of testosterone undecanoate therapy on LUTS in men with $\mathrm{LOH}$

In a series of papers, we have reported the effects of testosterone administration on a number of variables relating to the ailments of the aging male. The studies were not specifically designed to investigate the effects of testosterone administration to elderly men on symptoms of LUTS, but effects of testosterone treatment on the International Prostate Symptoms Score (IPSS) were recorded. In the first study, the effects of administration of parenteral testosterone undecanaote (TU) over 12 months were analyzed [53]. There were positive clinical effects of administration of TU on the IPSS and also on parameters of the metabolic syndrome, progressive over the 12 month study period. Since the effects were progressive over the 12 months of the study, it is likely that the effects take time to occur following testosterone administration. In the second study the effects of testosterone gel in a dose of $50 \mathrm{mg} /$ day over 9 months on symptoms of $\mathrm{LOH}$ were compared to those of parenteral TU [54]. The higher plasma levels of $\mathrm{T}$ generated with TU than with T gel $(50 \mathrm{mg} /$ day $)$ were more effective in reducing the scores on the IPSS, probably indicating that there is a relationship between plasma levels of testosterone and their effects on LUTS. The third study investigated the effects testosterone gel in a dose of $50 \mathrm{mg} /$ day over 9 months which had a positive effect on scores of the IPSS. Subsequently, these men shifted their testosterone treatment to administration of parenteral testosterone undecanaote (TU) [54). There were positive clinical effects of administration of $\mathrm{T}$ gel on the IPSS and also on parameters of the metabolic syndrome, and there was a significant further improvement of the IPSS when TU was administered after 9 months of administration of $\mathrm{T}$ gel when plasma $\mathrm{T}$ levels increased to higher levels than with $\mathrm{T}$ gel.

It is obvious that better designed placebo-controlled, randomized, studies are needed to explore the potential relationship between $\mathrm{T}$ and LUTS and the potential of a therapeutic effect of $\mathrm{T}$ in cases of $\mathrm{T}$ deficiency. It is important to uncover whether there is a threshold effect, so men with a range of baseline $\mathrm{T}$ levels should be included. Further, the effect of $\mathrm{T}$ in men with distinctly different IPSS should be investigated for a potential salutary effect of $T$ administration. It would also be significant to explore whether normalization of plasma $\mathrm{T}$ has an adjunctive therapeutic effect to the more established pharmacological treatment modalities of LUTS. Urodynamic assessment may provide greater insight into the potential mode of action of $\mathrm{T}$ on LUTS. The potential beneficial effects of $\mathrm{T}$ on LUTS may well be indirect, and therefore sufficiently powered studies are required to convincingly demonstrate an effect of T on LUTS.

\section{Conclusions}

It is common for aging men to experience urinary problems subsumed under the umbrella term LUTS. LUTS is an important determinant of quality of life [55]. Several other ailments of the aging male, such as erectile dysfunction and the metabolic syndrome appear to be related to circulating levels of testosterone. At an epidemiological level this relationship has been more difficult to demonstrate for LUTS and the relationship between LUTS and circulating levels of testosterone may be indirect. The relationship between the metabolic syndrome and LUTS may be based on the fact that the metabolic syndrome is associated with an over- 
activity of autonomic nervous system [9]. This overactivity of the autonomic nervous system may play a key role in increasing the severity of LUTS above an intrinsic basal intensity that is determined by the genitourinary anatomical/pathophysiological characteristics of other ailments leading to LUTS $[12,13]$.

At present, the targets for a potentially beneficial effect of testosterone on the urinary system can be only sketchily described,

Some studies investigating the effects of restoration of plasma testosterone levels in elderly men to normal found a positive effect on variables of the metabolic syndrome and scores of the IPSS. The evidence that testosterone treatment has a beneficial effect on LUTS must be regarded as preliminary, but in view of the impact LUTS has on the lives of elderly men [55], this relationship is worthy of further investigation.

Conflict of interest statement Farid Saad is an employee of BayerSchering Pharma Ag, manufacturer of testosterone undecanoate.

Open Access This article is distributed under the terms of the Creative Commons Attribution Noncommercial License which permits any noncommercial use, distribution, and reproduction in any medium, provided the original author(s) and source are credited.

\section{References}

1. Rohrmann S, Nelson WG, Rifai N, Kanarek N, Basaria S, Tsilidis KK, Smit E, Giovannucci E, Platz EA (2007) Serum sex steroid hormones and lower urinary tract symptoms in third national health and nutrition examination survey (NHANES III). Urology 69:708-713

2. Kaufman JM, Vermeulen A (2005) The decline of androgen levels in elderly men and its clinical and therapeutic implications. Endocr Rev 26:833-876

3. El-Sakka AI, Hassoba HM (2006) Age related testosterone depletion in patients with erectile dysfunction. J Urol 176:2589-2593

4. Schatzl G, Madersbacher S, Temml C, Krenn-Schinkel K, Nader A, Sregi G, Lapin A, Hermann M, Berger P, Marberger M (2003) Serum androgen levels in men: impact of health status and age. Urology 61:629-633

5. Litman HJ, Bhasin S, O'Leary MP, Link CL, McKinlay JB (2007) An investigation of the relationship between sex-steroid levels and urological symptoms: results from the Boston Area Community Health survey. BJU Int 100:321-326

6. Koritsiadis G, Stravodimos K, Mitropoulos D, Doumanis G, Fokitis I, Koritsiadis S, Constantinides C (2008) Androgens and bladder outlet obstruction: a correlation with pressure-flow variables in a preliminary study. BJU Int 101:1542-1546 (E-pub)

7. Zitzmann M, Faber S, Nieschlag E (2006) Association of specific symptoms and metabolic risks with serum testosterone in older men. J Clin Endocrinol Metab 91:4335-4343

8. Zitzmann M (2007) Mechanisms of disease: pharmacogenetics of testosterone therapy in hypogonadal men. Nat Clin Pract Urol 4:161-166

9. Bjorntorp P, Rosmond R (2000) The metabolic syndrome: a neuroendocrine disorder? Br J Nutr 83(suppl 1):S49-S57

10. Rosmond R, Dallman MF, Bjorntorp P (1998) Stress-related cortisol secretion in men: relationships with abdominal obesity and endocrine, metabolic and hemodynamic abnormalities. J Clin Endocrinol Metab 83:1853-1859

11. McVary KT, Rademaker A, Lloyd GL, Gann P (2005) Autonomic nervous system overactivity in men with lower urinary tract symptoms secondary to benign prostatic hyperplasia. J Urol 174:13271433

12. Kasturi S, Russell S, McVary KT (2006) Metabolic syndrome and lower urinary tract symptoms secondary to benign prostatic hyperplasia. Curr Urol Rep 7:288-292

13. Ullrich PM, Lutgendorf SK, Kreder KJ (2007) Physiologic reactivity to a laboratory stress task among men with benign prostatic hyperplasia. Urology 70:487-491 discussion 491-482

14. Rohrmann S, De Marzo AM, Smit E, Giovannucci E, Platz EA (2005) Serum C-reactive protein concentration and lower urinary tract symptoms in older men in the Third National Health and Nutrition Examination Survey (NHANES III). Prostate 62:27-33

15. Teoh H, Verma S (2007) C-reactive protein, metabolic syndrome, and end organ damage. Metabolism 56:1620-1622

16. El-Sakka AI, Sayed HM, Tayeb KA (2007) Type 2 diabetes-associated androgen alteration in patients with erectile dysfunction. Int J Androl 18 Sep [Epub ahead of print] PMID: 17877718

17. Renehan AG, Frystyk J, Flyvbjerg A (2006) Obesity and cancer risk: the role of the insulin-IGF axis. Trends Endocrinol Metab 17:328-336

18. Hammarsten J, Hogstedt B (2001) Hyperinsulinaemia as a risk factor for developing benign prostatic hyperplasia. Eur Urol 39:151-158

19. Yialamas MA, Dwyer AA, Hanley E, Lee H, Pitteloud N, Hayes FJ (2007) Acute sex steroid withdrawal reduces insulin sensitivity in healthy men with idiopathic hypogonadotropic hypogonadism. J Clin Endocrinol Metab 92:4254-4259

20. El-Sakka AI (2006) Lower urinary tract symptoms in patients with erectile dysfunction: analysis of risk factors. J Sex Med 3:144-149

21. Rosen RC (2006) Update on the relationship between sexual dysfunction and lower urinary tract symptoms/benign prostatic hyperplasia. Curr Opin Urol 16:11-19

22. McVary K (2006) Lower urinary tract symptoms and sexual dysfunction: epidemiology and pathophysiology. BJU Int 97(suppl 2):23-28 discussion 44-25

23. Yassin A, Saad F, Hoesl CE, Traish AM, Hammadeh M, Shabsigh $\mathrm{R}$ (2006) Alpha-adrenoceptors are a common denominator in the pathophysiology of erectile function and BPH/LUTS: implications for clinical practice. Andrologia 38:1-12

24. El-Sakka AI (2005) Lower urinary tract symptoms in patients with erectile dysfunction: is there a vascular association? Eur Urol 48:319-325

25. Kaplan SA, Meehan AG, Shah A (2006) The age related decrease in testosterone is significantly exacerbated in obese men with the metabolic syndrome: what are the implications for the relatively high incidence of erectile dysfunction observed in these men? J Urol 176:1524-1527 discussion 1527-1528

26. Kapoor D, Malkin CJ, Channer KS, Jones TH (2005) Androgens, insulin resistance and vascular disease in men. Clin Endocrinol (Oxf) 63:239-250

27. Isidori AM, Giannetta E, Greco EA, Gianfrilli D, Bonifacio V, Isidori A, Lenzi A, Fabbri A (2005) Effects of testosterone on body composition, bone metabolism and serum lipid profile in middle-aged men: a meta-analysis. Clin Endocrinol (Oxf) 63:280-293

28. Shabsigh R, Perelman MA, Lockhart DC, Lue TF, Broderick GA (2005) Health issues of men: prevalence and correlates of erectile dysfunction. J Urol 174:662-667

29. Allan CA, Strauss BJ, Burger HG, Forbes EA, McLachlan RI (2007) Testosterone therapy prevents gain in visceral adipose tissue and loss of skeletal muscle in non-obese aging men. J Clin Endocrinol Metab 93:139-146 
30. Rosenzweig BA, Bolina PS, Birch L, Moran C, Marcovici I, Prins GS (1995) Location and concentration of estrogen, progesterone, and androgen receptors in the bladder and urethra of the rabbit. Neurourol Urodyn 14:87-96

31. Keast JR (1999) The autonomic nerve supply of male sex organsan important target of circulating androgens. Behav Brain Res 105:81-92

32. Watkins TW, Keast JR (1999) Androgen-sensitive preganglionic neurons innervate the male rat pelvic ganglion. Neuroscience 93:1147-1157

33. Hall R, Andrews PL, Hoyle CH (2002) Effects of testosterone on neuromuscular transmission in rat isolated urinary bladder. Eur $\mathrm{J}$ Pharmacol 449:301-309

34. Juan YS, Onal B, Broadaway S, Cosgrove J, Leggett RE, Whitbeck C, De E, Sokol R, Levin RM (2007) Effect of castration on male rabbit lower urinary tract tissue enzymes. Mol Cell Biochem 301:227-233

35. Filippi S, Morelli A, Sandner P, Fibbi B, Mancina R, Marini M, Gacci M, Vignozzi L, Vannelli GB, Carini M, Forti G, Maggi M (2007) Characterization and functional role of androgen-dependent PDE5 activity in the bladder. Endocrinology 148:1019-1029

36. McVary KT (2006) Unexpected insights into pelvic function following phosphodiesterase manipulation: what's next for urology? Eur Urol 50:1153-1156

37. Ehren I, Adolfsson J, Wiklund NP (1994) Nitric oxide synthase activity in the human urogenital tract. Urol Res 22:287-290

38. Smet PJ, Jonavicius J, Marshall VR, de Vente J (1996) Distribution of nitric oxide synthase-immunoreactive nerves and identification of the cellular targets of nitric oxide in guinea-pig and human urinary bladder by cGMP immunohistochemistry. Neuroscience 71:337-348

39. Qiu Y, Kraft P, Craig EC, Liu X, Haynes-Johnson D (2001) Identification and functional study of phosphodiesterases in rat urinary bladder. Urol Res 29:388-392

40. Werkstrom V, Svensson A, Andersson KE, Hedlund P (2006) Phosphodiesterase 5 in the female pig and human urethra: morphological and functional aspects. BJU Int 98:414-423

41. Chamness SL, Ricker DD, Crone JK, Dembeck CL, Maguire MP, Burnett AL, Chang TS (1995) The effect of androgen on nitric oxide synthase in the male reproductive tract of the rat. Fertil Steril 63:1101-1107

42. Truss MC, Stief CG, Uckert S, Becker AJ, Wefer J, Schultheiss D, Jonas U (2001) Phosphodiesterase 1 inhibition in the treatment of lower urinary tract dysfunction: from bench to bedside. World $\mathbf{J}$ Urol 19:344-350

43. Sairam K, Kulinskaya E, McNicholas TA, Boustead GB, Hanbury DC (2002) Sildenafil influences lower urinary tract symptoms. BJU Int 90:836-839
44. Montorsi F, Corbin J, Phillips S (2004) Review of phosphodiesterases in the urogenital system: new directions for therapeutic intervention. J Sex Med 1:322-336

45. Uckert S, Hedlund P, Andersson KE, Truss MC, Jonas U, Stief CG (2006) Update on phosphodiesterase (PDE) isoenzymes as pharmacologic targets in urology: present and future. Eur Urol 50:1194-1207 discussion 1207

46. Mulhall JP, Guhring P, Parker M, Hopps C (2006) Assessment of the impact of sildenafil citrate on lower urinary tract symptoms in men with erectile dysfunction. J Sex Med 3:662-667

47. McVary KT, Monnig W, Camps JL Jr, Young JM, Tseng LJ, van den Ende G (2007) Sildenafil citrate improves erectile function and urinary symptoms in men with erectile dysfunction and lower urinary tract symptoms associated with benign prostatic hyperplasia: a randomized, double-blind trial. J Urol 177:1071-1077

48. Andersson KE, Uckert S, Stief C, Hedlund P (2007) Phosphodiesterases (PDEs) and PDE inhibitors for treatment of LUTS. Neurourol Urodyn 26:928-933

49. Holmang S, Marin P, Lindstedt G, Hedelin H (1993) Effect of long-term oral testosterone undecanoate treatment on prostate volume and serum prostate-specific antigen concentration in eugonadal middle-aged men. Prostate 23:99-106

50. Mskhalaya G, Rozhivanov, RV, Nesterov, MN, Kalinchenko, SY (2006) The efficiency and safety of human chorionic gonadotropin (HCG) therapy on low urinary tract symptoms (LUTS) in men with benign prostatic hyperplasia (BPH). In: Fifth congress of the International Society for the Study of the Aging Male. Salzburg, Austria

51. Karazindiyanoğlu S, Çayan S (2007) Beneficial effects of testosterone administration of bladder function. In: European Association of Urology, Berlin, Germany

52. Mskhalaya G, Tishova JA, Koval AN, Vishnevskiy EL, Kalinchenko SY (2007) The efficiency of testosterone undecanoat (Nebido) therapy on lower urinary tract symptoms (LUTS) in men with late-onset hypogonadism (LOH). In: 1st European congress of the Society for the Study of the Aging Male. Warsaw, Poland

53. Saad FG LJ, Haider A, Yassin A (2007) An exploratory study of the effects of 12 month administration of the novel long-acting testosterone undecanoate on measures of sexual function and the metabolic syndrome. Arch Androl 53:353-357

54. Saad F, Gooren LJ, Haider A, Yassin A (2008) A dose-response study of testosterone on sexual dysfunction and features of the metabolic syndrome using testosterone gel and parenteral testosterone undecanoate. J Androl 29:102-105

55. Robertson C, Link CL, Onel E, Mazzetta C, Keech M, Hobbs R, Fourcade R, Kiemeney L, Lee C, Boyle P, McKinlay JB (2007) The impact of lower urinary tract symptoms and comorbidities on quality of life: the BACH and UREPIK studies. BJU Int 99:347-354 